\title{
Fixed Point Theorems in Relational Metric Spaces with an Application to Boundary Value Problems
}

\author{
PRASAD Gopi $^{1, *}$ and KHANTWAL Deepak ${ }^{2}$ \\ ${ }^{1}$ Department of Mathematics, HNB Garhwal University, Srinagar Garhwal, India. \\ 2 Department of Mathematics, Graphic Era Hill University, Dehradun, India.
}

Received 5 July 2020; Accepted 4 November 2020

\begin{abstract}
In this paper, we establish fixed point theorems for generalized nonlinear contractive mappings using the concept of $w$-distance in relational metric spaces. Thus we generalize the recent results of Senapati and Dey [J. Fixed Point Theory Appl. 19, 2945-2961 (2017)] and many other important results relevant to this literature. In order to revel the usefulness of such investigations, an application to first order periodic boundary value problem are given. Moreover, we furnish a non-trivial example to demonstrate the validity of our generalization over previous existing results.
\end{abstract}

AMS Subject Classifications: 47H10, 54H25

Chinese Library Classifications: O175.27

Key Words: Binary relation; $\mathcal{R}$-lower semi-continuity; relational metric spaces.

\section{Introduction}

The classical Banach contraction principle (Bcp) has many inferences and huge applicability in mathematical theory and because of this, Bcp has been improved and generalized in various metric settings. One such interesting and important setting is to establish fixed point results in metric spaces equipped with an arbitrary binary relation. Utilizing the notions of various kind of binary relations such as partial order, strict order, near order, tolerance etc. on metric spaces, many researcher are doing their research during several years (see [1-16]) and attempting to obtain new extensions of the celebrated Bcp. Among these extensions, we must quote the one due to Alam and Imdad [8], where some relation theoretic analogues of standard metric notions (such as continuity and completeness)

*Corresponding author. Email addresses: gopiprasad127@gmail . com (G. Prasad), deepakkhantwal15@gmail . com (D. Khantwal) 
were used. Further, Ahmadullah et al. [14] extended the above setting for nonlinear contractions and obtained an extension of the Boyd-Wong [17] fixed point theorem to such spaces.

On the other hand, recently Senapati and Dey [11] improved and refined the main result of Alam and Imdad [8], Ahmadullah et al. [14] and many others, by utilizing the notion of $w$-distance in relational metric spaces, that is, metric spaces endowed with an arbitrary binary relation. Moreover, for further motivation of research in this direction, we refer some important recent generalizations of $w$-distance with applications to boundary value problem as well (see, e.g., [19-21]). It is our aim in this paper to give an extension of these results to nonlinear $\varphi$-contraction and explore the possibility of their application in finding a solution of first order periodic boundary value problem too.

\section{Preliminaries}

Throughout this chapter, $\mathcal{R}$ stands for a non-empty binary relation, $\mathbb{N}_{0}$ stands for the the set of whole numbers, i.e., $\mathbb{N}_{0}=\mathbb{N} \cup\{0\}$ and $R$ for the set of all real numbers.

Definition 2.1. ([8]). Let $\mathcal{R}$ be a binary relation on a non-empty set $X$ and $x, y \in X$. We say that $x$ and $y$ are $\mathcal{R}$-comparative if either $(x, y) \in \mathcal{R}$ or $(y, x) \in \mathcal{R}$. We denote it by $[x, y] \in \mathcal{R}$.

Definition 2.2. ([8]). Let $X$ be a non-empty set and $\mathcal{R}$ a binary relation on $X$. A sequence $\left\{x_{n}\right\} \subset X$ is called an $\mathcal{R}$-preserving if $\left(x_{n}, x_{n+1}\right) \in \mathcal{R}$ for all $n \in \mathbb{N}_{0}$.

Definition 2.3. ([8]). Let $X$ be a non-empty set and $T$ a self-mapping on $X$. A binary relation $\mathcal{R}$ on $X$ is called $T$-closed if for any $x, y \in X,(x, y) \in \mathcal{R}$ implies $(T x, T y) \in \mathcal{R}$.

Definition 2.4. ([14]). Let $(X, d)$ be a metric space and $\mathcal{R}$ a binary relation on $X$. We say that $(X, d)$ is $\mathcal{R}$-complete if every $\mathcal{R}$-preserving Cauchy sequence in $X$ converges.

The following notion is a generalization of $d$-self-closedness of a partial order relation ( $)$ (defined by Turinici [5-6]).

Definition 2.5. $([8])$. Let $(X, d)$ be a metric space. A binary relation $\mathcal{R}$ on $X$ is called $d$-selfclosed if for any $\mathcal{R}$-preserving sequence $\left\{x_{n}\right\}$ such that $x_{n} \stackrel{d}{\longrightarrow} x$, there exists a subsequence $\left\{x_{n_{k}}\right\}$ of $\left\{x_{n}\right\}$ with $\left[x_{n_{k}}, x\right] \in \mathcal{R}$ for all $k \in \mathbb{N}_{0}$.

Definition 2.6. ([14]). Let $(X, d)$ be a metric space, $\mathcal{R}$ a binary relation on $X$ and $x \in X$. A self-mapping $T$ on $X$ is called $\mathcal{R}$-continuous at $x$ if for any $\mathcal{R}$-preserving sequence $\left\{x_{n}\right\}$ such that $x_{n} \stackrel{d}{\longrightarrow} x$, we have $T\left(x_{n}\right) \stackrel{d}{\longrightarrow} T(x)$. Moreover, $T$ is called $\mathcal{R}$-continuous if it is $\mathcal{R}$-continuous at each point of $X$.

The notion of $\mathcal{R}$-lower semi-continuity (briefly, $\mathcal{R}$-LSC) of a function is defined by Senapati and Dey [11] as follows: 
Definition 2.7. Let $(X, d)$ be a metric space and $R$ be a binary relation defined on $X$. A function $f: X \rightarrow R \cup\{-\infty,+\infty\}$ is said to be $\mathcal{R}$-LSC at $x$ if for every $\mathcal{R}$-preserving sequence $x_{n}$ converging to $x$, we have $\liminf _{n \rightarrow+\infty} f\left(x_{n}\right) \geq f(x)$.

By presenting examples the respective authors explained that the $\mathcal{R}$-LSC is weaker than $\mathcal{R}$-continuity as well as lower semi-continuity (see for details [11]) and modify the definition of w-distance ( Definition 2.8) and the corresponding Lemma 1 presented in [18] in the context of metric spaces endowed with an arbitrary binary relation $\mathcal{R}$ as follows:

Definition 2.8. Let $(X, d)$ be a metric space and $\mathcal{R}$ be a binary relation on $X$. A function $p$ : $X \times X \rightarrow[0,+\infty)$ is said to be a $w$-distance on $X$ if

$\left(w_{1}\right) \quad p(x, z) \leq p(x, y)+p(y, z)$ for any $x, y, z \in X$;

$\left(w_{2}\right)$ for any $x \in X, p(x,):. X \rightarrow[0, \infty)$ is $\mathcal{R}$-lower semi-continuous;

$\left(w_{3}\right)$ for any $\epsilon>0$, there exists $\delta>0$, such that $p(z, x) \leq \delta$ and $p(z, y) \leq \delta$ imply $d(x, y) \leq \epsilon$.

Let $\Phi$ be the family of all mappings $\varphi:[0,+\infty) \rightarrow[0,+\infty)$ satisfying the following properties

1. $\varphi$ is increasing;

2. $\sum_{n=1}^{+\infty} \varphi^{n}(t)<\infty$ for each $t>0$, where $\varphi^{n}$ is the $n$-th iterate of $\varphi$.

Recall that, the necessary condition of any real convergent series $\sum_{n} a_{n}$ is that

$$
\lim _{n \rightarrow+\infty} a_{n}=0 .
$$

The following two lemmas are required in our subsequent discussion.

Lemma 2.1. ([14]). Let $\varphi \in \Phi$. Then for all $t>0$, we have $\varphi(t)<t$.

Lemma 2.2. ([11]). Let $(X, d)$ be a metric space endowed with binary relation $\mathcal{R}$ and $p: X \times$ $X \rightarrow[0,+\infty)$ be a w-distance. Suppose $\left(x_{n}\right)$ and $\left(y_{n}\right)$ are two $\mathcal{R}$-preserving sequences in $X$ and $x, y, z \in X$. Let $\left(u_{n}\right)$ and $\left(v_{n}\right)$ be sequences of positive real numbers converging to 0 . Then, we have the followings:

$\left(L_{1}\right)$ If $p\left(x_{n}, y\right) \leq u_{n}$ and $p\left(x_{n}, z\right) \leq v_{n}$ for all $n \in \mathbb{N}$, then $y=z$. Moreover, if $p(x, y)=0$ and $p(x, z)=0$, then $y=z$.

$\left(L_{2}\right)$ If $p\left(x_{n}, y_{n}\right) \leq u_{n}$ and $p\left(x_{n}, z\right) \leq v_{n}$ for all $n \leq N$, then $y_{n} \rightarrow z$.

$\left(L_{3}\right)$ If $p\left(x_{n}, x_{m}\right) \leq u_{n}$ for all $m>n$, then $\left(x_{n}\right)$ is an $\mathcal{R}$-preserving Cauchy sequence in $X$. 
$\left(L_{4}\right)$ If $p\left(x_{n}, y\right) \leq u_{n}$ for all $n \in \mathbb{N}$, then $\left(x_{n}\right)$ is an $\mathcal{R}$-preserving Cauchy sequence in $X$.

Given a binary relation $\mathcal{R}$ and a self-mapping $T$ on a nonempty set $X$, we use the following notations :

(i) $F(T):=$ the set of all fixed points of $T$,

(ii) $X(T, \mathcal{R}):=\{x \in X:(x, T x) \in \mathcal{R}\}$.

\section{Main Results}

In this section, we first consider the existence of fixed points for mappings in relational metric spaces.

Theorem 3.1. Let $(X, d)$ be a metric space with a w-distance ' $p$ ' and a binary relation ' $\mathcal{R}$ ' on $X$. Let $T$ be a self-mapping on $X$ satisfying the following assumptions :

(a) there exists $Y \subseteq X$ with $T(X) \subseteq Y$ such that $(Y, d)$ is $\mathcal{R}$-complete,

(b) $\mathcal{R}$ is $T$-closed,

(c) either $T$ is $\mathcal{R}$-continuous or $\mathcal{R}_{\mid Y}$ is d-self-closed,

(d) $X(T, \mathcal{R})$ is non-empty,

(e) there exists $\varphi \in \Phi$ such that

$$
p(T x, T y) \leq \varphi(p(x, y),
$$

for all $x, y \in X$ with $(x, y) \in \mathcal{R}$. Then $T$ has a fixed point.

Proof. In the light of assumption (d), let $x_{0}$ be an arbitrary element of $X(T, \mathcal{R})$. Define a sequence $\left\{x_{n}\right\}$ of Picard iterates with initial point $x_{0}$, i.e,

$$
x_{n}=T^{n}\left(x_{0}\right) \quad \text { for all } n \in \mathbb{N}_{0} .
$$

Since $\left(x_{0}, T x_{0}\right) \in \mathcal{R}$ and $\mathcal{R}$ is $T$-closed, we have

$$
\left(T x_{0}, T^{2} x_{0}\right) \quad\left(T^{2} x_{0}, T^{3} x_{0}\right) \cdots\left(T^{n} x_{0}, T^{n+1} x_{0}\right), \cdots \in \mathcal{R},
$$

so that

$$
\left(x_{n}, x_{n+1}\right) \in \mathcal{R} \quad \text { for all } n \in \mathbb{N}_{0} .
$$

Thus the sequence $\left\{x_{n}\right\}$ is $\mathcal{R}$-preserving. Applying the contractive condition (e), we have

$$
p\left(x_{n}, x_{n+1}\right)=p\left(T x_{n-1}, T x_{n}\right) \leq \varphi\left(p\left(x_{n-1}, x_{n}\right)\right) \text { for all } n \in \mathbb{N}_{0} .
$$


By mathematical induction and the property $\left(\Phi_{1}\right)$, we obtain $p\left(x_{n}, x_{n+1}\right) \leq \varphi^{n}\left(p\left(x_{0}, x_{1}\right)\right)$, for all $n \in \mathbb{N}_{0}$. Now, for all $m, n \in \mathbb{N}_{0}$ with $m \geq n$, we have

$$
\begin{aligned}
p\left(x_{n}, x_{m}\right) & \leq p\left(x_{n}, x_{n+1}\right)+p\left(x_{n+1}, x_{n+2}\right)+\ldots+p\left(x_{m-1}, x_{m}\right) \\
& \leq \varphi^{n}\left(p\left(x_{0}, x_{1}\right)\right)+\varphi^{n+1}\left(p\left(x_{0}, x_{1}\right)\right)+\ldots+\varphi^{m-1}\left(p\left(x_{0}, x_{1}\right)\right) \\
& =\sum_{k=n}^{m-1} \varphi^{k}\left(p\left(x_{0}, x_{1}\right)\right) \leq \sum_{k \geq n} \varphi^{k}\left(p\left(x_{0}, x_{1}\right)\right) \\
& \rightarrow 0 \quad \text { as } n \rightarrow+\infty .
\end{aligned}
$$

Therefore, by $\left(L_{3}\right)$, of Lemma 2.2 we have $\left\{x_{n}\right\}$ is an $\mathcal{R}$-preserving Cauchy sequence in $Y$. As $(Y, d)$ is an $\mathcal{R}$-complete, we must have $x_{n} \rightarrow x$ as $n \rightarrow+\infty$ for some $x \in Y$.

Next we claim that $x$ is a fixed point of $T$. At first, we consider that $T$ is $\mathcal{R}$-continuous.

Since $\left\{x_{n}\right\}$ is an $\mathcal{R}$-preserving sequence with $x_{n} \stackrel{d}{\longrightarrow} x, \mathcal{R}$-continuity of $T$ implies that

$$
x_{n+1}=T x_{n} \stackrel{d}{\longrightarrow} T x \text {. }
$$

Using the uniqueness of the limit, we obtain $T x=x$, i.e, $x$ is a fixed point of $T$.

Alternately, let us assume that $\mathcal{R}_{\mid Y}$ is $d$-self-closed. So there exists a subsequence $\left\{x_{n_{k}}\right\}$ of $\left\{x_{n}\right\}$ with $\left[x_{n_{k}}, x\right] \in \mathcal{R}$ for all $k \in \mathbb{N}_{0}$. By using the fact that $\left[x_{n_{k}}, x\right] \in \mathcal{R}$, contractive assumption (e) and $\mathcal{R}$-lower-semi-continuity of $p$, we have

$$
p\left(x_{n_{k}}, x\right) \leq \liminf _{k \rightarrow+\infty} p\left(x_{n_{k}}, x_{n_{k}+m}\right) \leq \liminf _{k \rightarrow+\infty} \sum_{i \geq n_{k}} \varphi^{i} p\left(x_{0}, x_{1}\right)=0 .
$$

Since $\mathcal{R}$ is $T$-closed and $\left(x_{n_{k}}, x\right) \in \mathcal{R}$, so

$$
p\left(T x_{n_{k}}, T x\right) \leq \varphi\left(p\left(x_{n_{k}}, x\right) \leq \varphi\left(\liminf _{k \rightarrow \infty} p\left(x_{n_{k}}, x_{n_{k}+m}\right)\right)=0 .\right.
$$

Finally, owing to condition $\left(L_{1}\right)$ of Lemma 2.2 , we must have $T x=x$, i.e., $x$ is a fixed point of $T$.

\subsection{Uniqueness result}

We state the uniqueness related result as follows :

Theorem 3.2. In addition to the hypotheses of Theorem 3.1, suppose that any of the assumptions $\left(u_{1}\right)$ or $\left(u_{2}\right)$ holds :

$\left(u_{1}\right)$ For every $x, y \in T(X)$ there exists $z \in T(X)$ such that $(z, x),(z, y) \in \mathcal{R}$.

$\left.\left(u_{2}\right) \mathcal{R}\right|_{T(X)}$ is complete.

Then $T$ has a unique fixed point. 
Proof. In addition to the hypotheses of Theorem 3.1, suppose that condition $\left(u_{1}\right)$ hold. Then, for any two fixed points $x, y$ of $T$, there exists an element $z \in T(X)$, such that

$$
(z, x) \in \mathcal{R} \text { and }(z, y) \in \mathcal{R} .
$$

Since $\mathcal{R}$ is $T$-closed, we have

$$
\left(T^{n}(z), x\right) \in \mathcal{R} \quad \text { and } \quad\left(T^{n}(z), y\right) \in \mathcal{R} \quad \text { for all } n \in \mathbb{N}_{0} .
$$

Applying contractive condition (e), we have

$$
\begin{aligned}
& p\left(T^{n}(z), x\right)=p\left(T^{n}(z), T^{n} x\right) \leq \varphi^{n}(p(z, x)), \\
& p\left(T^{n}(z), y\right)=p\left(T^{n}(z), T^{n} y\right) \leq \varphi^{n}(p(z, y)) .
\end{aligned}
$$

Let us consider $u_{n}=\varphi^{n}(p(z, x))$ and $v_{n}=\varphi^{n}(p(z, y))$. Clearly, $\left\{u_{n}\right\}$ and $\left\{v_{n}\right\}$ are two sequences of real numbers converging to 0 . Hence, by $\left(L_{1}\right)$ of Lemma 2.2, we have $x=y$, i.e., $T$ has a unique fixed point.

Secondly, suppose that in addition to the hypotheses of Theorem 3.1 condition $\left(u_{2}\right)$ hold. Suppose $x, y$ are any two fixed points of $T$. Then we must have $(x, y) \in \mathcal{R}$ or $(y, x) \in$ $\mathcal{R}$. For $(x, y) \in \mathcal{R}$, we have

$$
p(x, y)=p(T(x), T(y)) \leq \varphi(p(x, y))<p(x, y)
$$

which is a contradiction. Hence, we must have $x=y$. Similarly, if $(y, x) \in \mathcal{R}$, we have $x=y$.

Example 3.1. Let $X=[0,+\infty)$ equipped with usual metric $d$. Then $(X, d)$ is a complete metric space. Define a binary relation $(x, y) \in \mathcal{R}$ implies $x \preceq y$ on $X$ and the mapping $T: X \rightarrow X$ by

$$
T(x)=\frac{x}{1+x} \quad \text { for all } x \in X
$$

Then $\mathcal{R}$ is $T$-closed. Define $\varphi:[0, \infty) \rightarrow[0, \infty)$ by $\varphi(t)=\frac{t}{1+t}$ for all $t \in[0, \infty)$, and a $w$ distance $p: X \times X \rightarrow X$ by $p(x, y)=y$. Now for all $x, y \in X$ with $(x, y) \in \mathcal{R}$, we have

$$
p(T(x), T(y))=p\left(\frac{x}{1+x}, \frac{y}{1+y}\right) \leq \varphi(p(x, y)), \quad \frac{y}{1+y} \leq \varphi(y)=\frac{y}{1+y^{\prime}}
$$

so that $T$ and $\varphi$ satisfy assumption (e) of Theorem 3.1. Observe that all the other conditions of Theorem 3.1 are also satisfied. Therefore, $T$ has a unique fixed point (namely $x=0)$.

Remark 3.1. It is interesting to note that the mapping $T$ in above example does not satisfy the contractive condition of Theorem 2.1 in Senapati and Dey [11]. For example, if we consider $x=0$ and $y=\epsilon$ where $\epsilon$ is arbitrary small but positive. Clearly, $(0, \epsilon) \in \mathcal{R}$ and if we take a constant $\lambda$ such that $p(T(x), T(y)) \leq \lambda p(x, y)$, i.e. $\frac{\epsilon}{1+\epsilon} \leq \lambda \epsilon$ then $\lambda \geq \frac{1}{1+\epsilon}$ which amounts to say that $\lambda \geq 1$ so that $\lambda \notin[0,1)$. Thus Example 3.1 vindicate the utility of Theorem 3.1 over the results of Sanapati and Dey [11] and many others. 
Remark 3.2. If we take $\varphi(t)=\lambda t$, in our main result Theorem 3.1, then we obtain the Theorem 2.1 of Senapati and Dey [11] and if we set $p(x, y)=d(x, y)$, and $\varphi(t)=\lambda t$, in our main result, we obtain the Theorem 3.1 of Alam and Imdad [8]. Hence our main result is an improved and generalized version of relation-theoretic metrical fixed-point theorems of Alam and Imdad [8], Senapati and Dey [11] and many others.

\section{An application}

As an application, we present a unique solution for the first order periodic boundary value problem equipped with an arbitrary binary relation, wherein our main results are applicable. We consider the following first order periodic boundary value problem:

$$
x^{\prime}(t)=f(t, x(t)) ; \quad t \in I=[0, T] ; \quad x(0)=x(T),
$$

where $T>0$ and $f: I \times R \rightarrow R$ is a continuous function.

Let $C(I)$ denote the space of all continuous functions defined on $I$. We recall the following definitions.

Definition 4.1. ([9]). A function $\alpha \in C^{1}(I)$ is called a lower solution of (4.1), if

$$
\begin{aligned}
& \alpha^{\prime}(t) \leq f(t, \alpha(t)), \quad t \in I, \\
& x(0) \leq x(T) .
\end{aligned}
$$

Definition 4.2. ([9]). A function $\alpha \in C^{1}(I)$ is called a upper solution of (4.1), if

$$
\begin{aligned}
& \alpha^{\prime}(t) \geq f(t, \alpha(t)), \quad t \in I, \\
& x(0) \geq x(T) .
\end{aligned}
$$

Theorem 4.1. In addition to the problem (4.1), suppose that there exist $\lambda>0$ such that for all $x, y \in R$ with $x \leq y$.

$$
0 \leq f(t, y)+\lambda y-[f(t, x)+\lambda x] \leq \lambda \varphi(y-x) .
$$

Then the existence of a lower solution or an upper solution of problem (4.1) ensures the existence and uniqueness of a solution of problem (4.1).

Proof. Problem (4.1) can be rewritten as

$$
x^{\prime}(t)+\lambda x(t)=f(t, x(t))+\lambda x(t) ; \quad t \in I=[0, T] ; \quad x(0)=x(T) .
$$

This problem is equivalent to the integral equation

$$
x(t)=\int_{0}^{T} G(t, s)[f(s, x(s))+\lambda x(s)] d s
$$


where

$$
G(t, s)= \begin{cases}\frac{e^{\lambda(T+s-t)}}{e^{\lambda T}-1}, & 0 \leq s<t \leq T, \\ \frac{e^{\lambda(s-t)}}{e^{\lambda T}-1}, & 0 \leq t<s \leq T .\end{cases}
$$

Define a mapping $T: C(I) \rightarrow C(I)$ by

$$
(T x)(t)=\int_{0}^{T} G(t, s)[f(s, x(s))+\lambda x(s)] d s,
$$

and a binary relation

$$
\mathcal{R}=\{(x, y) \in C(I) \times C(I): x(t) \leq y(t) \text { for all } t \in I\} .
$$

(i) Note that $C(I)$ equipped with the sup-metric, i.e., $d(x, y)=\sup |x(t)-y(t)|$ for $t \in I$ and $x, y \in C(I)$ is complete metric space and hence $(C(I), d)$ is $\mathcal{R}$-complete.

(ii) Choose an $\mathcal{R}$-preserving sequence $\left\{x_{n}\right\}$ such that $x_{n} \stackrel{d}{\longrightarrow} z$. Then for all $t \in I$, we get

$$
x_{0}(t) \leq x_{1}(t) \leq \cdots \leq x_{n}(t) \leq x_{n+1}(t) \leq \cdots
$$

and convergence to $x(t)$ implies that $x_{n}(t) \leq z(t)$ for all $t \in I, n \in \mathbb{N}_{0}$, which amounts to saying that $\left[x_{n}, z\right] \in \mathcal{R}$ for all $n \in \mathbb{N}_{0}$. Hence, $\mathcal{R}$ is $d$-self-closed.

(iii) For any $(x, y) \in \mathcal{R}$, i.e. $x(t) \leq y(t)$ then by (4.2), we have

$$
f(t, x(t))+\lambda x(t) \leq f(t, y(t))+\lambda y(t) \quad \text { for all } t \in I
$$

and $G(t, s)>0$ for $(t, s) \in I \times I$, we have

$$
\begin{aligned}
(T x)(t) & =\int_{0}^{T} G(t, s)[f(s, x(s))+\lambda x(s)] d s, \\
& \leq \int_{0}^{T} G(t, s)[f(s, y(s))+\lambda y(s)] d s \\
& =(T y)(t) \quad \text { for all } t \in I,
\end{aligned}
$$

which implies that $(T x, T y) \in \mathcal{R}$, i.e., $\mathcal{R}$ is $T$-closed.

(iv) Let $\alpha \in C^{1}(I)$ be a lower solution of (4.1), then we must have

$$
\alpha^{\prime}(t)+\lambda \alpha(t) \leq f(t, \alpha(t))+\lambda \alpha(t) \quad \text { for all } t \in I .
$$

Multiplying both sides by $e^{\lambda t}$, we have

$$
\left(\alpha(t) e^{\lambda t}\right)^{\prime} \leq[f(t, \alpha(t))+\lambda \alpha(t)] e^{\lambda t} \quad \text { for all } t \in I
$$


which implies that

$$
\alpha(t) e^{\lambda t} \leq \alpha(0)+\int_{0}^{t}[f(s, \alpha(s))+\lambda \alpha(s)] e^{\lambda s} d s \quad \text { for all } t \in I .
$$

As $\alpha(0) \leq \alpha(T)$, we have

$$
\alpha(0) e^{\lambda T} \leq \alpha(T) e^{\lambda T} \leq \alpha(0)+\int_{0}^{T}[f(s, \alpha(s))+\lambda \alpha(s)] e^{\lambda s} d s
$$

therefore

$$
\alpha(0) \leq \int_{0}^{T} \frac{e^{\lambda s}}{e^{\lambda T}-1}[f(s, \alpha(s))+\lambda \alpha(s)] d s .
$$

By using (4.3) and (4.4), we have

$$
\begin{aligned}
\alpha(t) e^{\lambda t} & \leq \int_{0}^{T} \frac{e^{\lambda s}}{e^{\lambda T}-1}[f(s, \alpha(s))+\lambda \alpha(s)] d s+\int_{0}^{t} e^{\lambda s}[f(s, \alpha(s))+\lambda \alpha(s)] d s \\
& =\int_{0}^{t} \frac{e^{\lambda(T+s)}}{e^{\lambda T}-1}[f(s, \alpha(s))+\lambda \alpha(s)] d s+\int_{t}^{T} \frac{e^{\lambda(s)}}{e^{\lambda T}-1}[f(s, \alpha(s))+\lambda \alpha(s)] d s,
\end{aligned}
$$

so that

$$
\begin{aligned}
\alpha(t) & \leq \int_{0}^{t} \frac{e^{\lambda(T+s-t)}}{e^{\lambda T}-1}[f(s, \alpha(s))+\lambda \alpha(s)] d s+\int_{t}^{T} \frac{e^{\lambda(s-t)}}{e^{\lambda T}-1}[f(s, \alpha(s))+\lambda \alpha(s)] d s \\
& =\int_{0}^{T} G(t, s)[f(s, \alpha(s))+\lambda \alpha(s)] d s \\
& =(T \alpha)(t)
\end{aligned}
$$

for all $t \in I$, i.e., $(\alpha(t), T \alpha(t)) \in \mathcal{R}$ for all $t \in I$ which implies that $X(T, \mathcal{R}) \neq \phi$.

(v) For all $(x, y) \in \mathcal{R}$,

$$
\begin{aligned}
d(T x, T y) & =\sup _{t \in I}|(T x)(t)-(T y)(t)|=\sup _{t \in I}((T y)(t)-(T x)(t)) \\
& \leq \sup _{t \in I} \int_{0}^{T} G(t, s)[f(s, y(s))+\lambda y(s)-f(s, x(s))-\lambda x(s)] d s \\
& \leq \sup _{t \in I} \int_{0}^{T} G(t, s) \lambda \varphi(y(s)-x(s)) d s \\
& =\lambda \varphi(d(x, y)) \sup _{t \in I} \int_{0}^{T} G(t, s) d s \\
& =\lambda \varphi(d(x, y)) \sup _{t \in I} \frac{1}{e^{\lambda T}-1}\left(\left.\frac{1}{\lambda} e^{\lambda(T+s-t)}\right|_{0} ^{t}+\left.\frac{1}{\lambda} e^{\lambda(s-t)}\right|_{t} ^{T}\right)
\end{aligned}
$$




$$
\begin{aligned}
& =\lambda \varphi(d(x, y)) \sup _{t \in I} \frac{1}{\lambda\left(e^{\lambda T}-1\right)}\left(e^{\lambda T}-1\right) \\
& =\varphi(d(x, y)),
\end{aligned}
$$

so that

$$
d(T x, T y) \leq \varphi(d(x, y)) .
$$

Now, if we set $p(x, y)=d(x, y)$, then we have

$$
p(T x, T y) \leq \varphi(p(x, y)) \quad \forall x, y \in C(I) \text { such that }(x, y) \in \mathcal{R}, x
$$

where $\varphi \in \Phi$. Hence all the conditions of Theorem 3.1 are satisfied, consequently $T$ has a fixed point. Finally following the proof of our earlier Theorem 3.2, $T$ has a unique fixed point, which is in fact a unique solution of the problem (4.1).

\section{Acknowledgement}

The authors thank the referees for their careful reading of the manuscript and useful comments.

\section{References}

[1] Turinici M., Abstract comparison principles and multi-variable Gronwall-Bellman inequalities. J. Math. Anal. Appl. 117 (1), (1986), 100-127.

[2] Ran A. C. M. and Reurings M. C. B., A fixed point theorem in partially ordered sets and some applications to matrix equations. Proc. Amer. Math. Soc. 132 (5) (2004), 1435-1443.

[3] Nieto J. J. and Rodriguez-Lopez R., Contractive mapping theorems in partially ordered sets and applications to ordinary differential equations. Order 22 (3), (2005), 223-239.

[4] Harjani J. and Sadarangani K., Fixed point theorems for weakly contractive mappings in partially ordered sets. Nonlinear Anal. 71 (2009), 3403-3410.

[5] Turinici M., Ran and Reuring's theorems in ordered metric spaces. J. Indian Math. Soc. 78 (2011), 207-214.

[6] Turinici M. , Nieto-Lopez theorems in ordered metric spaces. Math. Student 81 (4), (2012), 219-229.

[7] Samet B. and Turinici M., Fixed point theorems on a metric space endowed with an arbitrary binary relation and applications. Commun. Math. Anal. 13 (2), (2012), 82-97.

[8] Alam A. and Imdad M., Relation-theoretic contraction principle. J. Fixed Point Theory Appl. 17 (4) (2015), 693-702.

[9] Kutbi M. A., Alam A. and Imdad M., Sharpening some core theorems of Nieto and Rodriguez-Lopez with application to boundary value problem. Fixed Point Theory Appl. 2015, 198 (2015).

[10] Alam A. and Imdad M., Monotone generalized contractions in ordered metric spaces. Bull. Korean Math. Soc. 53 (1) (2016), 61-81.

[11] Senapati T. and Dey L. K. , Relation-theoretic metrical fixed point results via $w$-distance with applications. J. Fixed Point Theory Appl. 19 (2017), 2945-2961. 
[12] Prasad G. and Dimri R. C., Fixed point results for weakly contractive mappings in relational metric spaces with an application. J. Anal. 26 (1), (2018), 151-162.

[13] Prasad G. and Dimri R. C. , Coincidence theorems in new generalized metric spaces under locally g-transitive binary relation. J. Indian Math. Soc. 85 (3-4), (2018), 396-410.

[14] Ahmadullah M., Imdad M. and Gubran R., Relation-theoretic metrical fixed point theorems under nonlinear contractions. Fixed Point Theory 20 (1) (2019), 3-18.

[15] Prasad G., Dimri R. C. and Bartwal A., Fixed points of Suzuki contractive mappings in relational metric spaces. Rend. Circ. Mat. Palermo, II, Ser. 69 (3), (2020), 1347-1358.

[16] Prasad G. , Fixed points of Kannan contractive mappings in relational metric spaces. J. Anal. (2020). https://doi.org/10.1007/s41478-020-00273-7

[17] Boyd D.W., and Wong J.S., On nonlinear contractions. Proc. Amer. Math. Soc. 20 (1969), 458464.

[18] Kada O., Suzuki T. and Takahashi W., Nonconvex minimization theorems and fixed point theorems in complete metric spaces. Math. Japonica. 44 (2) (1996), 381-391.

[19] Kostić A., Rakočević V. and Radenović S., Best proximity points involving simulation functions with $w_{0}$-distance. RACSAM. 113 (2019), 715-727.

[20] Thenmozhi S., Marudai M., Radenović S., Existence of positive solution for the eighth-order boundary value problem by Leray-Schauder alternative fixed point theorem. Axioms 8 (4) (2019), 13 pp, Article ID 129.

[21] Fabiano N., Nikolić N., Shanmugam T., Radenović S. and Čitaković N., Tenth order boundary value problem solution existence by fixed point theorem. J. Inequal. Appl. 2020 (1) (2020), $11 p p$. 